\title{
Three-dimensional ultrasound is increasing our knowledge of the fetus little by little
}

Three-dimensional ultrasound has been available for more than 10 years. The use of three-dimensional ultrasound appears to provide us with great benefits in fetal diagnosis; it allows the visualization of fetal malformation in three orthogonal planes at the same time; the stored volume can be rotated easily and dissected step by step from any of three spatial planes; and surface images enable us to give an effective presentation of fetal anomalies to parents and doctors. However, almost all detectable anomalies of the fetus can be detected by conventional twodimensional ultrasound. In two-dimensional ultrasound, experienced examiners can form an adequate threedimensional mental impression of fetal anomalies. So, has fetal diagnosis really been improved by the development of three-dimensional ultrasound? Is its benefit to fetal medicine limited to improvement in the presentation of fetal anomalies?

It is doubtful whether three-dimensional ultrasound has markedly extended the repertoire of fetal diagnosis. Our knowledge of the fetus, however, is certainly increasing little by little as we continue to use three-dimensional ultrasound daily in fetal examinations. For example, surface rendering of the fetal face enhances the visualization of fetal facial expressions. Before three-dimensional ultrasound was introduced for fetal examinations, we had already known of fetal facial movements: yawning, sucking, and swallowing. The use of three-dimensional ultrasound started to make us aware of the presence of various fetal facial expressions: smiling, crying, and grimacing, although the facial expressions may be only subjective impressions on the part of the examiner. It is, nevertheless, surprising that the development of facial expressions commences in fetal life, because facial expressions are essential elements of social behavior. Considering that fetal movements such as breathing movements are a sort of training in preparation for independent life after birth, I wonder whether facial expressions are necessary for the neonate to survive immediately after birth. Although the neonate has an independent life, the human neonate is highly dependent on the mother, because it is immature compared to neonates of other mammalian species. I therefore believe that social behaviors such as facial expressions are essential for the human neonate to survive, and the fetus is programmed to develop facial expressions in preparation for bonding with its parents after birth.

Similarly, we had already known of changes in fetal body growth before the introduction of three-dimensional ultrasound, but we have started to notice changes in the shape of the fetal body, from a muscular type in the second trimester to a plump type in the third trimester on threedimensional ultrasound. This change may be related to the growth of various parts of the body: musculoskeletal development, fat deposits, and the development of various internal organs. We will need to investigate each of these topics in the future.

Moreover, spatiotemporal image correlation (STIC) gives us three-dimensional moving images of the fetal heart and great vessels, enhancing our understanding of the characteristics of the fetal circulatory system. For example, the anatomical relationship between the heart and ascending aorta can be clearly demonstrated using STIC with the inversion mode; this modality shows that the ascending aorta bends sharply forward in relation to the four-chamber plane of the heart after it ascends for a short distance from the left ventricle.

Although recent three-dimensional ultrasound machines are highly sophisticated, three-dimensional ultrasound of the fetus poses some problems that need to be resolved before this examination method can be considered reliable. First, if the fetus is in contact with the placenta or the uterine wall, especially in oligohydramnios, it is difficult to provide an adequate surface reconstruction. Second, when the field of interest is far from the probe or the umbilical cord, and a fetal leg or arm is interposed between the probe and the structure concerned, the image quality of the fetal surface is markedly lowered, because the quality of surface reconstruction depends on the quality of the original data. Finally, artifacts can be produced by the movement of the probe or the fetus during the storage of volume data.

The fetus is still a mystery. We have just taken a glance at part of the fetal world. Although there are some prob- 
lems associated with three-dimensional ultrasound, we have certainly started to accumulate knowledge of the fetus by using this technique. Two-dimensional ultrasound opened the door to the fetus; now three-dimensional ultrasound will push us into the fetal world.

Shiro KOZUMA

Editor 